\title{
AiM \\ Effect of acupuncture on salivary immunoglobulin $A$ after a bout of intense exercise
}

Yuichi Matsubara, Kazuhiro Shimizu, Yuko Tanimura, et al.

Acupunct Med 2010 28: 28-32

doi: 10.1136/aim.2009.001677

Updated information and services can be found at:

http://aim.bmj.com/content/28/1/28.full.html

These include:

References This article cites 30 articles, 2 of which can be accessed free at: http://aim.bmj.com/content/28/1/28.full.html\#ref-list-1

Article cited in:

http://aim.bmj.com/content/28/1/28.full.html\#related-urls

Email alerting Receive free email alerts when new articles cite this article. Sign up in the service box at the top right corner of the online article.

\section{Notes}

To request permissions go to:

http://group.bmj.com/group/rights-licensing/permissions

To order reprints go to:

http://journals.bmj.com/cgi/reprintform

To subscribe to BMJ go to:

http://journals.bmj.com/cgi/ep 


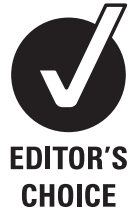

'Graduate School of Comprehensive Human Sciences, University of Tsukuba, Ibaraki, Japan

${ }^{2}$ Faculty of Sport Sciences,

Waseda University, Tokorozawa

Saitama, Japan

${ }^{3}$ Laboratory of Regenerative

Medical Engineering, Center for

Disease Biology and Integrative

Medicine, Graduate School of

Medicine, The University of

Tokyo, Tokyo, Japan

${ }^{4}$ Institute for Biomedical

Engineering, Consolidated

Research Institute for Advanced

Science and Medical Care,

Waseda University, Tokyo,

Japan

\section{Correspondence to}

Dr Ichiro Kono, Graduate School of Comprehensive Human

Sciences, University of Tsukuba,

Tennodai 1-1-1, Tsukuba, Ibaraki 305-8577, Japan; kono@

taiiku.tsukuba.ac.jp

Accepted 18 January 2010

\title{
Effect of acupuncture on salivary immunoglobulin A after a bout of intense exercise
}

\author{
Yuichi Matsubara, ${ }^{1}$ Kazuhiro Shimizu, ${ }^{1,2}$ Yuko Tanimura, ${ }^{1}$ Toshikazu Miyamoto, ${ }^{1}$ \\ Takayuki Akimoto, ${ }^{3,4}$ Ichiro Kono $^{1}$
}

\begin{abstract}
In the field of athletics, acupuncture has been used for treatment of injury, reduction of fatigue and management of physical condition. However, there is little information on the effect of acupuncture on the immune function in response to exercise.
\end{abstract}

Purpose The aim of this study was to examine the effect of acupuncture treatment on the mucosal immune function after a single period of intense exercise by measuring salivary immunoglobulin A (SlgA).

Methods 12 healthy men (23.6 \pm SEv 0.3 years) participated in this study with a crossover design. The subjects exercised on a bicycle equipped with an ergometer at $75 \% \mathrm{VO}_{2} \max$ for $60 \mathrm{~min}$. Acupuncture treatment was applied at LU6, LI4, ST36 and ST6, for 30 min after the exercise. The control treatment was rest without acupuncture and that the order of the treatment was randomised. We measured parameters including saliva flow rate, SlgA concentration, SlgA secretion rate, heart rate and plasma catecholamine concentration all before the exercise and at $1 \mathrm{~h}, 2 \mathrm{~h}, 3 \mathrm{~h}, 4 \mathrm{~h}$ and $24 \mathrm{~h}$ after the exercise. The visual analogue scale for self-perceived tiredness and the profile of mood states questionnaires were recorded before the exercise and at $24 \mathrm{~h}$ after the exercise.

Result Intense exercise-induced decrease of SlgA levels was attenuated by the acupuncture treatment. In contrast, the subjective fatigue score and psychological measurement were not affected by the acupuncture. Conclusion Acupuncture treatment may attenuate the decrease in SlgA level induced by intense exercise.

\section{INTRODUCTION}

Athletes have to maintain and even improve highfitness levels and skills to perform well at competitions. Therefore, they need to do high-frequency, high-intensity training. Among elite athletes and coaches it is well known that athletes have a higher risk of upper respiratory tract infection (URTI) than non-athletes. ${ }^{1}$ It has been reported that acute highintensity exercise and long-term heavy training lead to a decrease in immune functions..$^{2-5}$ Additionally, Nieman has reported that people who habitually do moderate exercise have less risk of URTI than those people who do not; however, he also has reported that high-intensity exercise increases the risk of URTI. ${ }^{6}$ Since it is known that URTI adversely affects training and performance, ${ }^{7}$ it is important for athletes to avoid URTI in order to maintain a good physical and mental condition. ${ }^{89}$

Several studies have shown that suppression of salivary immunoglobulin $\mathrm{A}(\mathrm{SIg} \mathrm{A})$ level in elite athletes frequently results in URTI. ${ }^{10} 11$ SIgA, which is the predominant immunoglobulin in secretions of the mucosal immune system, penetrates the body through the mucosal tissues, ${ }^{12} 13$ neutralises various toxins and viruses, and inhibits the attachment and replication of pathogenic microorganisms. ${ }^{13} 14$

Acupuncture is used most commonly as an adjunctive treatment for the relief of chronic or acute pain. Acupuncture treatment is also a very common method for modulating the physical condition of various athletes. ${ }^{15}$ In athletics, acupuncture is most widely used to treat pain associated with musculoskeletal injuries, but it has also been used to treat physical conditions, such as relieving pain and tiredness, and promote regeneration of injured areas of the body. Furthermore, it has been reported that acupuncture treatment is effective for soothing tight muscles, improving peripheral blood circulation, increasing the pain threshold and adjusting the autonomic nervous system. ${ }^{16-18}$ Acupuncture also has been recognised to increase 'natural healing power,' including immunological responses that can improve the physical and mental condition and even can cure and prevent diseases. ${ }^{19}$

Although acupuncture treatment is considered to adjust the immune function and contribute to the stabilisation of human homeostasis, ${ }^{20}$ few studies have been conducted to determine the combined effects of acupuncture treatment and exercise on the human immune function. So far, our group has published only one study investigating the effect of acupuncture treatment on the immune function of athletes during competition. ${ }^{21}$ We reported that continuous acupuncture treatment counteracted intense training-induced decrease in SIgA and also had positive effects on physical and mental conditions. This study was the first to demonstrate that acupuncture treatment attenuated the reduction in the mucosal immune function of athletes during competition. Since this study was conducted during a period of sports competition, it could serve as a basic experiment for a new study, one that investigates the capability of acupuncture treatment to attenuate both the acute intense exercise-induced decrease in SIgA and the reduction in the mucosal immune function in athletes. The only conceptual change necessary in the experiment would be to omit the factors other than acupuncture treatment that affect SIgA levels.

The purpose of this study was to examine the effect of acupuncture treatment on the mucosal immune function after acute high-intensity exercise by measuring salivary SIgA levels. We hypothesised that acupuncture treatment would lessen the decrease of SIgA induced by the exercise. 
Table 1 Subjects' characteristics

\begin{tabular}{lc}
\hline Number & 12 \\
Age (year) & $23.6 \pm 0.3$ \\
Height $(\mathrm{cm})$ & $171.4 \pm 1.6$ \\
Body mass (kg) & $66.0 \pm 2.4$ \\
Body fat (\%) & $16.2 \pm 1.1$ \\
Maximal oxygen uptake (ml/kg/min) & $41.6 \pm 1.6$ \\
\hline
\end{tabular}

Date were expressed mean \pm SE

\section{METHODS \\ Subjects}

Twelve healthy sedentary men $(23.6 \pm 0.3$ years) were recruited from a collegiate population to participate in this study (table 1). None of subjects reported a history of smoking. All subjects were provided verbal and written information about the purpose, measures and ethical considerations of the experiment before their informed written consent was obtained. This study was approved by the Ethical Committees of the Institute of Health and Sport Sciences and the Institute of Clinical Medicine of University of Tsukuba; it also was determined that the study conformed to the principles outlined in the Declaration of Helsinki.

The subjects were randomly distributed into two groups and with crossover design. The experiment was divided into two phases, each beginning with a submaximal cycle exercise test. In the first phase, one group received acupuncture treatment while the other rested, and within a week, in the second phase, the group roles were reversed.

\section{Exercise test}

Maximal oxygen uptake $\left(\mathrm{VO}_{2} \mathrm{max}\right)$ of the subjects was determined through the use of an incremental bicycle exercise test. The subjects rode the bicycle to exhaustion, and their breath-bybreath oxygen uptake and carbon dioxide production were monitored on a cycle ergometor (AE280S; Minato Medical Science, Osaka, Japan). The protocol consisted of 2 min of unloaded pedalling and subsequent incremental exercise. The workload was increased to 60, 80 and $100 \mathrm{~W}$ for $2 \mathrm{~min}$. Subsequently, the workloads increased by $30 \mathrm{~W}$ every $3 \mathrm{~min}$ until exhaustion. Objective criteria for maximal effort included at least two of the following: (1) increased workload without corresponding increase in $\mathrm{VO}_{2}$; (2) respiratory exchange quotient equal to or greater than 1.10; (3) pedal cadence less than $50 \mathrm{rpm}$ in spite of maximal voluntary effort. The highest $\mathrm{O}_{2}$ uptake over a $30 \mathrm{~s}$ period was defined as $\mathrm{VO}_{2}$ max. Subjects then underwent a submaximal cycle exercise test. The tests were taken within 1 week of the $\mathrm{VO}_{2}$ max test, at least $4 \mathrm{~d}$ apart. In the submaximal cycle exercise test, subjects exercised on a cycle ergometer for $1 \mathrm{~h}$ at $75 \%$ of their $\mathrm{VO}_{2}$ max.

\section{Heart rates}

The heart rates of the subjects were measured at the same points of sample collections. The heart rate was measured by Polar S610 (Polar, Kempele, Finland).

\section{Subjective evaluations of physical and mental conditions}

Subjective evaluations of subject conditions were made during the experiment. The visual analogue scale for self-perceived tiredness was used at the same points as sample collections. ${ }^{22}$ This rating was assessed using a $100 \mathrm{~mm}$ continuous scale. In addition, a Japanese version of the profile of mood state (POMS) questionnaire was administered to assess the subjective mental states before the exercise test and after $24 \mathrm{~h} .{ }^{23}$ Six mood states are used in POMS:
T-A (tension), D (depression), A-H (anger), V (vigour), F (fatigue) and $\mathrm{C}$ (confusion). Subjects are given a score for each trait according to their responses to 65 statements. Each mood dimension was rated on a scale of 1 to 4 from 'not at all' to 'extremely.'

\section{Acupuncture treatment}

After the submaximal cycle exercise test, the acupuncture treatment was performed by an experienced acupuncturist, and the same points were used as in our previous study. ${ }^{21}$ The following bilateral points were used as acupuncture points: LU6 in the forearms, LI4 in the hands, ST36 in the legs and ST6 in both sides of the face. $^{24-27}$ After standard disinfecting of the insertion sites, disposable stainless needles $(50 \mathrm{~mm}$ long, $0.20 \mathrm{~mm}$ in diameter, SEIRIN, Shizuoka, Japan) were inserted through the skin to a depth of 5-10 $\mathrm{mm}$, and manipulated until the subjects felt a sensation from the needles. Electrodes were connected to three points (LU6, LI4 and ST36) and then attached to an electric stimulator (OhmPulser LFP4800; Zen Iryoki, Fukuoka, Japan). The points were electrically stimulated with a low frequency of $2 \mathrm{~Hz}$ for $30 \mathrm{~min}$. At the same time, the fourth point (ST6) was manually stimulated for $30 \mathrm{~min}$ until needle sensation was reached every $5 \mathrm{~min}$.

\section{Saliva collection}

Saliva samples were collected at rest before exercise (pre), immediately after exercise (P0), 1, 2, 3 and $4 \mathrm{~h}$ after exercise (P1, P2, P3, $\mathrm{P} 4)$, and $24 \mathrm{~h}$ after exercise (P24), as described in our previous studies. $^{28} 29$ The subjects were free to drink water during the experiment. Light meals were given to all the subjects at the sample collection, which was taken within $1 \mathrm{~h}$ after the exercise. The subjects rinsed their mouths for $30 \mathrm{sec} 3$ times with $100 \mathrm{ml}$ of mineral water, and then rested for at least $5 \mathrm{~min}$. Saliva production was stimulated by the chewing of sterilised cotton (Salivette; Saersted, Vümbrecht, Germany) for $2 \mathrm{~min}$ at a frequency of $1 \mathrm{chew} / \mathrm{sec}$. Saliva was separated from the cotton swab by centrifugation at $3000 \mathrm{rpm}$ for $15 \mathrm{~min}$. The amount of saliva in grammes was converted to millilitres based on an assumed saliva density of $1 \mathrm{~g} / \mathrm{ml}$, as described in one of our previous studies. ${ }^{28}$ After the sample volume was measured, samples were frozen at $-80^{\circ} \mathrm{C}$ and stored until analysis.

\section{SIgA measurement}

SIgA concentrations were measured by enzyme-linked immunosorbent assay, according to the procedures of our previous study. ${ }^{21}$ Rabbit antihuman secretory component IgG fraction (MBL, Nagoya, Japan) with coating buffer was added to each well of a microtitre plate (IMMULON-2, Dynex Technologies, Chantilly, VA) and kept at $4^{\circ} \mathrm{C}$ for more than $8 \mathrm{~h}$. After $250 \mu \mathrm{l}$ of phosphate-buffered saline (PBS) containing 1\% bovine serum albumin (BSA, Sigma, St Louis, MO) was added, the wells were blocked for $2 \mathrm{~h}$ at normal room temperature. Saliva samples were thawed, centrifuged at $10000 \mathrm{rpm}$ for $5 \mathrm{~min}$, diluted with $100 \mathrm{\mu l}$ of PBS containing 1\% BSA and incubated for $1 \mathrm{~h}$. By using purified human SIgA (MP Biomedicals, Inc, Santa Ana, CA), known concentrations of SIgA were also plated to establish standard values. After the plate was washed with PBS-Tween, goat Fab'anti-IgA conjugated with horse radish peroxide HRP (mannose binding lectin) was added to the plate and incubated for $1 \mathrm{~h}$. After washing, substrate solution was added, and the colour intensity produced after 10 min was measured by a microplate reader (Mode1550, Bio Rad, Hercules, CA) at $490 \mathrm{~nm}$ wave length. For analysis of the SIgA levels, data were expressed as the SIgA secretion rate $(\mu \mathrm{g} / \mathrm{min})$. This rate was calculated by 
multiplying the absolute SIgA concentration $(\mu \mathrm{g} / \mathrm{ml})$ by the saliva flow rate $(\mathrm{ml} / \mathrm{min})$, which was calculated by dividing the total volume of saliva obtained in each sample $(\mathrm{ml})$ by the time taken to produce the sample (min).

\section{Blood collection and measurement of plasma catecholamine concentrations}

Blood samples were collected at the same times as saliva collections. Blood was sampled from the antecubital vein in the arm. Plasma was generated by centrifuging blood at $3000 \mathrm{rpm}$ for $15 \mathrm{~min}$ at $4^{\circ} \mathrm{C}$; it was frozen at $-80^{\circ} \mathrm{C}$ until the analysis of catecholamine concentrations. Plasma concentrations of epinephrine and norepinephrine were measured by an automatic catecholamine analyser (HLC-725CA II, Tosoh, Tokyo, Japan).

\section{Statistical analysis}

All data were represented as mean \pm SE. The effect of acupuncture treatment was determined by using two-way analyses of variance with repeated measures. A Tukey-Kramer adjustment was applied to the posthoc test. The significance level of all statistical analyses was set at $\mathrm{p}<0.05$.

\section{RESULTS}

\section{Salivary SIgA levels}

As shown in figure 1, the SIgA secretion rate significantly decreased from P0 to P3 in the control group $(p<0.05)$ but did not decrease significantly in the acupuncture group. In contrast, the SIgA of the acupuncture group significantly increased at P24. The SIgA secretion rate was significantly higher in the acupuncture group than in the control group at P1 and P24 ( $<<0.05)$. The concentration of SIgA significantly decreased from P0 to P3 in the control group $(\mathrm{p}<0.05)$ and decreased significantly only at P2 in the acupuncture group (figure 2).

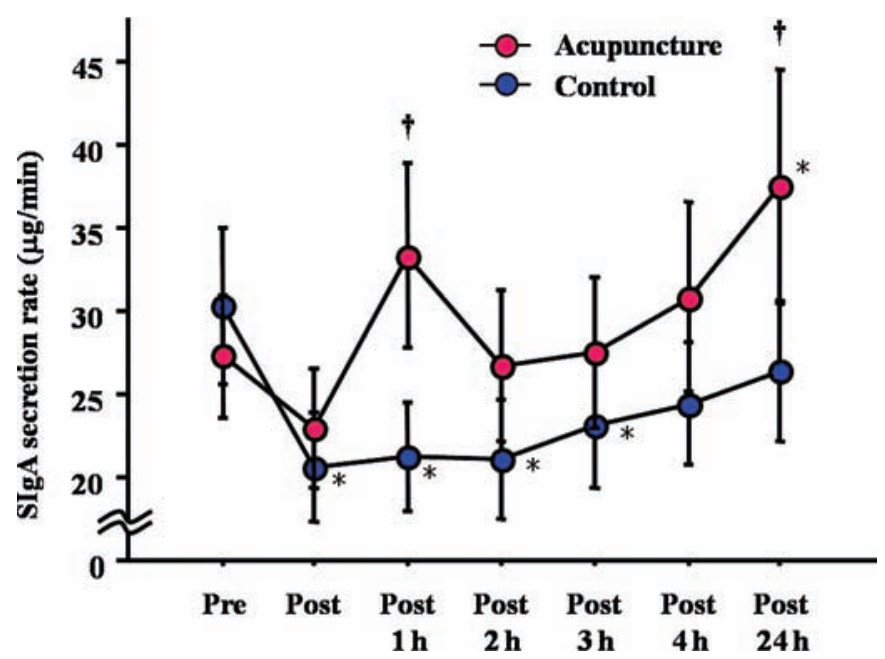

Figure 1 Temporal change in salivary immunoglobulin A (SIgA) secretion rate after exercise. Two-way analyses of variance (acupuncture-time) showed significant treatment effects in improving the levels of $\operatorname{SIgA}(p=0.0017)$. At P24, the SlgA secretion rate of the acupuncture group increased significantly. In the control group, the SIgA secretion rate significantly decreased from $\mathrm{P} 0$ to $\mathrm{P} 3$. Data were expressed as mean $\pm S E$; ${ }^{*} p<0.05$ (vs pre), $\uparrow p<0.05$ between groups

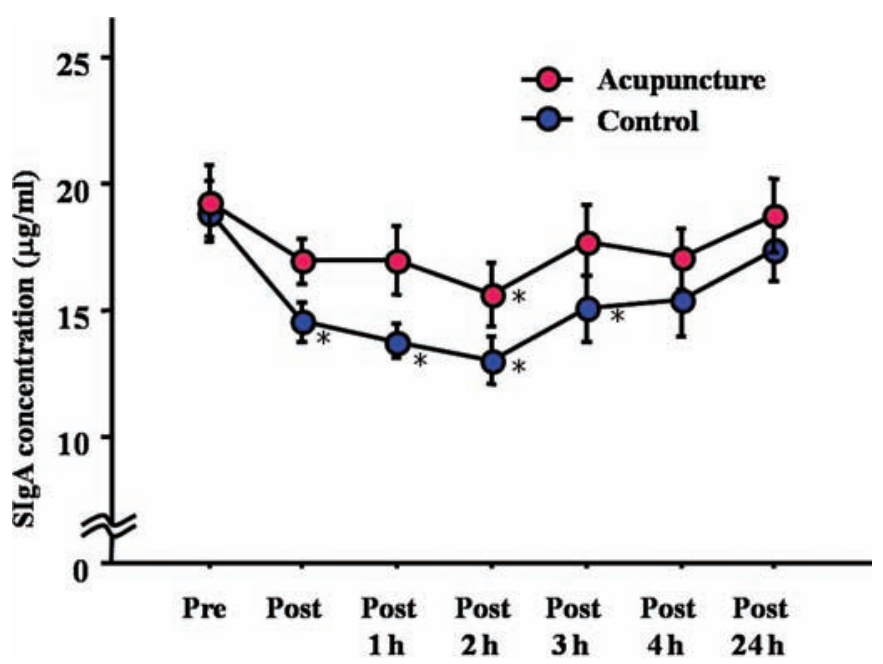

Figure 2 Temporal change in salivary immunoglobulin A (SIgA) concentration after exercise. Two-way analyses of variance (acupuncture-time) showed no significant treatment effects in improving the concentrations of $\operatorname{SIgA}(p=0.2647)$. However, there was a significant main effect of time for SIgA concentration $(p<0.001)$. In the acupuncture group, the SIgA concentration decreased significantly only at P2. In the control group, the SIgA concentration significantly decreased from $\mathrm{PO}$ to P3. Data were expressed as mean $\pm S E$; ${ }^{*} \mathrm{p}<0.05$ (vs pre).

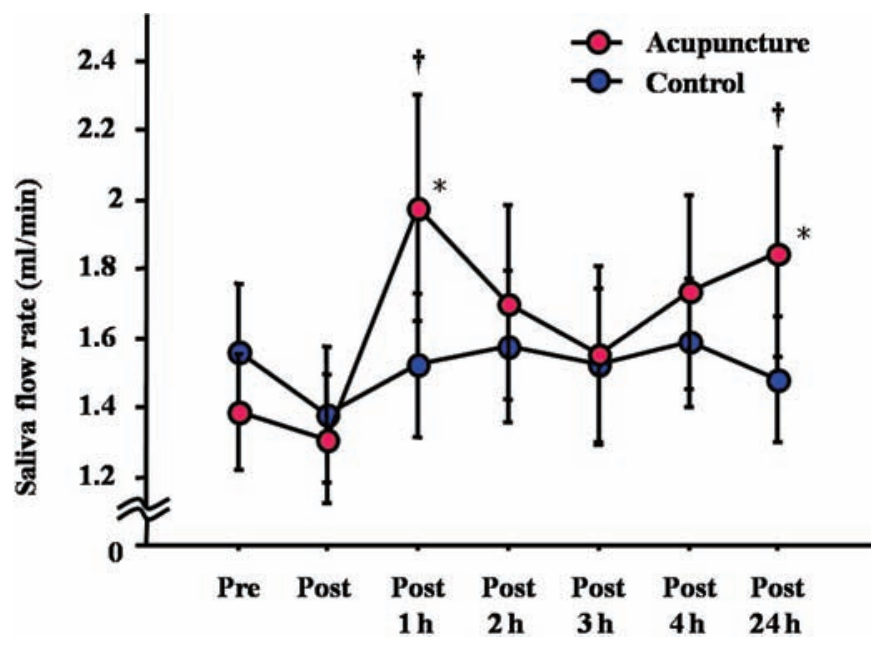

Figure 3 Temporal change in saliva flow rate after exercise. Two-way analyses of variance (acupuncture-time) showed significant treatment effects in increasing the saliva flow $(p=0.002)$. In the acupuncture group, the saliva flow rate significantly increased at P1 and P24. In the control group, the saliva flow rate did not significantly change during the study. Data were expressed as mean $\pm S E$; ${ }^{*} p<0.05$ (vs pre), $\uparrow p<0.05$ between groups.

\section{Saliva flow rates}

Figure 3 shows that the saliva flow rate did not significantly change in the control group. In contrast, the saliva flow rate of the acupuncture group significantly increased at P1 and P24 $(p<0.05)$. The saliva flow rate was significantly higher in the acupuncture group than in the control group at P1 and P24 $(p<0.05)$.

\section{Plasma catecholamine concentrations}

As shown in table 2, the epinephrine concentration increased at $\mathrm{P} 0$ and P1 in the control group. In contrast, the epinephrine concentration of the acupuncture group increased at P0. The norepi- 
Table 2 Temporal change in plasma levels of epinephrine and norepinephrine after the exercise

\begin{tabular}{|c|c|c|c|c|c|c|c|}
\hline & Pre & Post & Post $1 \mathrm{~h}$ & Post $2 \mathrm{~h}$ & Post $3 \mathrm{~h}$ & Post $4 \mathrm{~h}$ & Post 24 h \\
\hline \multicolumn{8}{|c|}{ Epinephrine (ng/ml) } \\
\hline Acupuncture & $0.03 \pm 0.01$ & $0.09 \pm 0.04^{*}$ & $0.04 \pm 0.02$ & $0.03 \pm 0.01$ & $0.03 \pm 0.01$ & $0.04 \pm 0.02$ & $0.04 \pm 0.02$ \\
\hline \multicolumn{8}{|c|}{ Norepinephrine (ng/ml) } \\
\hline Acupuncture & $0.22 \pm 0.08$ & $0.69 \pm 0.12^{*}$ & $0.30 \pm 0.09$ & $0.32 \pm 0.10^{*}$ & $0.32 \pm 0.09$ & $0.30 \pm 0.07$ & $0.29 \pm 0.07$ \\
\hline Control & $0.25 \pm 0.04$ & $0.69 \pm 0.24^{*}$ & $0.31 \pm 0.08$ & $0.37 \pm 0.14^{*}$ & $0.33 \pm 0.08^{*}$ & $0.30 \pm 0.05$ & $0.29 \pm 0.06$ \\
\hline
\end{tabular}

Date were expressed as mean \pm SE.

${ }^{*} \mathrm{p}<0.05$ (vs Pre)

Table 3 Temporal change in heart rate after the exercise

\begin{tabular}{|c|c|c|c|c|c|c|c|}
\hline & Pre & Post & Post $1 \mathrm{~h}$ & Post $2 \mathrm{~h}$ & Post $3 \mathrm{~h}$ & Post $4 \mathrm{~h}$ & Post $24 \mathrm{~h}$ \\
\hline \multicolumn{8}{|c|}{ Heart rate (beats/min) } \\
\hline Acupuncture & $57.0 \pm 2.1$ & $170.0 \pm .3 .5^{*}$ & $72.2 \pm 2.7^{*}$ & $74.8 \pm 2.2^{*}$ & $70.6 \pm 2.5^{*}$ & $65.8 \pm 2.8^{*}$ & $59.3 \pm 2.0$ \\
\hline Control & $61.0 \pm 3.3$ & $169.8 \pm 2.7^{*}$ & $75.8 \pm 3.0^{*}$ & $78.5 \pm 2.8^{*}$ & $74.7 \pm 2.4^{*}$ & $68.3 \pm 3.0$ & $60.3 \pm 1.9$ \\
\hline
\end{tabular}

Date were expressed as mean \pm SE.

* $\mathrm{p}<0.05$ (vs pre)

nephrine concentration increased at P0 and P2 in the control group. In contrast, the norepinephrine concentration of the acupuncture group increased at P0, P2 and P3.

\section{Heart rates}

Table 3 shows that the heart rate increased from P0 to P3 in the control group. In contrast, the heart rate of the acupuncture group increased from $\mathrm{P} 0$ to $\mathrm{P} 4$.

\section{Subjective evaluations of physical and mental conditions}

The visual analogue scale score on fatigue increased for both groups at $\mathrm{P} 0$ but returned to baseline after $\mathrm{P} 0$. All the mood states except 'vigour' at P24 in both groups were lower than they were at pre. However, there were no significant differences between the groups on any items.

\section{DISCUSSION}

In the present study, we provided evidence that acupuncture treatment positively affects SIgA levels and saliva flow rates in healthy sedentary male subjects after a single bout of intense exercise. This result suggests that acupuncture treatment works on the autonomic nervous system and may improve mucosal immune protection.

SIgA has the important function of blocking entrance of pathogenic organisms and preventing infections. It has been reported that reduction in SIgA levels might increase the risk of URTI. ${ }^{10} 3031$ It also has been reported that intensive exercise causes decrease of SIgA levels and increases the risk of URTI. ${ }^{1011}$ In the present study, we showed that acute highly intense exercise reduced SIgA levels, specifically that acupuncture treatment inhibited the reduction of SIgA levels and promoted recovery. This suggests that acupuncture treatment could be very effective in improving SIgA levels, reducing URTI, and maintaining better physical condition.

Secretion from the salivary glands is controlled by both the sympathetic and parasympathetic nervous systems. ${ }^{32}$ When the sympathetic nerve activity is dominant, small amounts of viscous saliva is secreted; when parasympathetic nerve activity is dominant, watery serous saliva is secreted. ${ }^{32}$ The mechanisms underlying the effects of acupuncture treatment still remain to be clarified, but many studies have shown that acupuncture treatment affected the autonomic nervous system. ${ }^{16}{ }^{17}$ Moreover, it has been reported that the autonomic nervous system reaction relate to central nerve system which stimulated by acupuncture. $^{33} 34$ This means that stimulating the sympathetic nerve, the parasympathetic nerve, or both nerves by acupuncture treatment could promote SIgA secretion. In this study, besides saliva, epinephrine, norepinephrine and heart rate, which are related to the autonomic nervous system, were measured. In the acupuncture group, the saliva flow rate increased at P1 and P24 but did not change in the control group throughout the study. On the other hand, the acupuncture treatment did not affect epinephrine, norepinephrine and heart rate whereas the intensive exercise did affect those factors. These results might suggest that the acupuncture treatment did not affect the whole autonomic nervous system, but only affected a part of it after intensive exercise. Therefore, it is possible that acupuncture treatment might improve SIgA secretion by stimulating the autonomic nervous system.

In this study, acute high-intensity exercise decreased SIgA levels, but then acupuncture treatment increased SIgA levels. However, the acupuncture treatment had no effect on the selfperceived tiredness resulting from the acute high-intensity exercise. There also were no effects on the POMS score in the acupuncture group. On the other hand, several studies have reported that acupuncture treatment promoted relief from muscle tension and tiredness among athletes. ${ }^{21}{ }^{35}$ It was possible that the subjects in this study did not receive continuous mental and physical stresses. It must be remembered, though, that SIgA levels were decreased even though the subjects were not aware of either mental or physical stress. The difference between subjective feelings and actual mucosal immune function might indicate one of the factors that cause the physical condition of athletes to deteriorate. These results suggest that the acupuncture treatment had little placebo influence on the subjects, because the subjective index did not change during the experiment.

It has been shown that acupuncture treatment restores the balance of the body's function. ${ }^{36}$ In addition, in actual acupuncture treatment, specific acupoints are selected based on each patient's individual conditions. In our study, the same acupuncture points were selected and the same treatments were given to 
all subjects, in order to keep all experimental conditions equal. Furthermore, the acupuncture treatment given in our study was physical stimulation by a needle, which might have a little different effect from that of a typical acupuncture treatment in clinics.

There is a need in the future to validate how effective acupuncture treatment is for decreasing URTI risks, by examining the relation between the influence of acupuncture treatment on SIgA levels and the incidence of URTI during a period of sports competition. There also is a need to define the mechanism by which acupuncture treatment promotes SIgA secretion. In addition, further research is needed to determine whether the effect of acupuncture was point specific in this study and whether electrical stimulation is necessary in the future. Once all the tasks mentioned above have been completed successfully, it is conceivable that acupuncture could be widely recognised as an effective treatment to keep athletes from getting URTI by adjusting their immune systems and even help athletes with their selfconditioning.

\section{Summary}

- Salivary immunoglobulin levels fall in athletes after a bout of exercise.

- Acupuncture immediately after exercise partially reverses this effect.

Acknowledgements This study was supported in part by a Grant in Aid for Scientific Research (20650111 and 19300228 to I. K.) and partly by 'Establishment of Consolidated Research Institute for Advanced Science and Medical Care', Encouraging Development of Strategic Research Centers Program, Special Coordination Funds for Promoting Science and Technology, Ministry of Education, Culture, Sports, Science and Technology, Japan. The authors also thank Visiting Professor Robert DiGiovanni (Waseda University, Japan) for his critical revision and editing of the manuscript.

Funding Ministry of Education, Culture, Sports, Science and Technology, Japan.

\section{Competing interests None.}

Provenance and peer review Not commissioned; externally peer reviewed.

Ethics approval This study was conducted with the approval of the Ethical Committees of the Institute of Health and Sport Sciences and the Institute of Clinical Medicine of University of Tsukuba.

Patient consent Obtained.

\section{REFERENCES}

1. Mackinnon LT. Chronic exercise training effects on immune function. Med Sci Sports Exerc 2000;32(7 Suppl):S369-76.

2. Fahlman MM, Engels HJ, Morgan AL, et al. Mucosal IgA response to repeated wingate tests in females. Int J Sports Med 2001;22:127-31.

3. Libicz S, Mercier B, Bigou N, et al. Salivary lgA response of triathletes participating in the French Iron Tour. Int J Sports Med 2006;27:389-94.

4. Pacque PF, Booth CK, Ball MJ, et al. The effect of an ultra-endurance running race on mucosal and humoral immune function. J Sports Med Phys Fitness 2007;47:496-501.

5. Pedersen BK, Ullum H. NK cell response to physical activity: possible mechanisms of action. Med Sci Sports Exerc 1994;26:140-6.

6. Nieman DC. Exercise, upper respiratory tract infection, and the immune system. Med Sci Sports Exerc 1994;26:128-39.

7. Pyne DB, Gleeson M. Effects of intensive exercise training on immunity in athletes. Int J Sports Med 1998;19(Suppl 3):S183-91;discussion S191-4.
8. Baxter C, Reilly T. Influence of time of day on all-out swimming. Br J Sports Med 1983:17:122-7.

9. Hickey MS, Costill DL, McConell GK, et al. Day to day variation in time trial cycling performance. Int J Sports Med 1992;13:467-70.

10. Gleeson M, McDonald WA, Pyne DB, et al. Salivary lgA levels and infection risk in elite swimmers. Med Sci Sports Exerc 1999;31:67-73.

11. Neville V, Gleeson M, Folland JP. Salivary IgA as a risk factor for upper respiratory infections in elite professional athletes. Med Sci Sports Exerc 2008;40:1228-36.

12. Lamm ME. Interaction of antigens and antibodies at mucosal surfaces. Annu Rev Microbiol 1997:51:311-40.

13. Mazanec MB, Nedrud JG, Kaetzel CS, et al. A three-tiered view of the role of IgA in mucosal defense. Immunol Today 1993;14:430-5

14. MacKinnon LT, Jenkins DG. Decreased salivary immunoglobulins after intense interval exercise before and after training. Med Sci Sports Exerc 1993;25:678-83.

15. Miyamoto T. Acupuncture treatment for muscle injury [in Japanese]. Jpn J Phys Fitness Sports Med 1997:43:39-41.

16. Haker E, Egekvist H, Bjerring P. Effect of sensory stimulation (acupuncture) on sympathetic and parasympathetic activities in healthy subjects. J Auton Nerv Syst 2000;79:52-9.

17. Knardahl S, Elam M, Olausson B, et al. Sympathetic nerve activity after acupuncture in humans. Pain 1998;75:19-25.

18. Sandberg M, Lindberg LG, Gerdle B. Peripheral effects of needle stimulation (acupuncture) on skin and muscle blood flow in fibromyalgia. Eur J Pain 2004;8:163-71.

19. Zijlstra FJ, van den Berg-de Lange I, Huygen FJ, et al. Anti-inflammatory actions of acupuncture. Mediators Inflamm 2003;12:59-69.

20. Kang Y, Li M, Yan W, et al. Electroacupuncture alters the expression of genes associated with lipid metabolism and immune reaction in liver of hypercholesterolemia mice. Biotechnol Lett 2007;29:1817-24.

21. Akimoto T, Nakahori C, Aizawa K, et al. Acupuncture and responses of immunologic and endocrine markers during competition. Med Sci Sports Exerc 2003;35:1296-302

22. Novas AM, Rowbottom DG, Jenkins DG. Tennis, incidence of URTI and salivary IgA. Int J Sports Med 2003;24:223-9.

23. Yokoyama K, Araki S, Kawakami N, et al. [Production of the Japanese edition of profile of mood states (POMS): assessment of reliability and validity]. Nippon Koshu Eisei Zasshi 1990;37:913-18.

24. Blom M, Dawidson I, Angmar-Månsson B. The effect of acupuncture on salivary flow rates in patients with xerostomia. Oral Surg Oral Med Oral Pathol 1992:73:293-8.

25. Blom M, Lundeberg T. Long-term follow-up of patients treated with acupuncture for xerostomia and the influence of additional treatment. Oral Dis 2000;6:15-24.

26. Dawidson I, Angmar-Mânsson B, Blom M, et al. Sensory stimulation (acupuncture) increases the release of calcitonin gene-related peptide in the saliva of xerostomia sufferers. Neuropeptides 1999;33:244-50.

27. Joos S, Schott C, Zou H, et al. Immunomodulatory effects of acupuncture in the treatment of allergic asthma: a randomized controlled study. J Altern Complement Med 2000;6:519-25

28. Akimoto $T$, Kumai $Y$, Akama $T$, et al. Effects of 12 months of exercise training on salivary secretory IgA levels in elderly subjects. Br J Sports Med 2003;37:76-9

29. Shimizu K, Kimura F, Akimoto T, et al. Effects of exercise, age and gender on salivary secretory immunoglobulin A in elderly individuals. Exerc Immunol Rev 2007;13:55-66.

30. Gleeson M, Pyne DB. Special feature for the Olympics: effects of exercise on the immune system: exercise effects on mucosal immunity. Immunol Cell Biol 2000;78:536-44.

31. Mackinnon LT, Hooper S. Mucosal (secretory) immune system responses to exercise of varying intensity and during overtraining. Int J Sports Med 1994; 15 Suppl 3:S179-83.

32. Garrett JR, Kidd A. The innervation of salivary glands as revealed by morphological methods. Microsc Res Tech 1993:26:75-91.

33. Dhond RP, Yeh C, Park K, et al. Acupuncture modulates resting state connectivity in default and sensorimotor brain networks. Pain 2008;136:407-18.

34. Hui KK, Marina 0, Claunch JD, et al. Acupuncture mobilizes the brain's default mode and its anti-correlated network in healthy subjects. Brain Res 2009;1287:84-103.

35. Lin JG, Yang SH. Effects of acupuncture on exercise-induced muscle soreness and serum creatine kinase activity. Am J Chin Med 1999;27:299-305.

36. Sung JJ. Acupuncture for gastrointestinal disorders: myth or magic. Gut 2002:51:617-19 